2021, Band 9, Heft 1/2

Seiten 179-184

zeitschrift-suburban.de

10.36900/suburban.v9i1/2.677

\title{
Lokale urbane Welten sichtbar machen
}

Kommentar zu Stefan Höhne und Boris Michel „Das Ende des Städtischen?

Pandemie, Digitalisierung und planetarische Enturbanisierung“

Stefan Höhne, Boris Michel ,Das Ende des Städtischen? Pandemie, Digitalisierung und planetarische Enturbanisierung'

Kommentare von:

Marcelo Lopes de Souza, Matthias Naumann, Anke Strüver, Markus Kip, Hannah Schilling, Roger Keil, Christian Haid, Anna-Lisa Müller, Nikolai Roskamm

Hannah Schilling

Im April vergangenen Jahres zirkulierten auf Twitter, in Zeitungen und im Fernsehen Bilder verwaister Stadtzentren. Die Welt auf Abstand (2020), so der Titel einer Dokumentation auf Arte, wurde zum Sinnbild. Die Nachfrage nach einem ,Haus im Grünen' stieg in Deutschland rasant an (Schäfer 2020), während Bürogebäude und Hotelkomplexe leer standen - die „Welt auf Abstand" scheint vor allem vom (groß-)städtischen Leben Abstand zu nehmen. In den entleerten Straßenzügen Berlins springen einem heute umso deutlicher Plakatwände ins Auge: In zentralen Einkaufsstraßen wie dem Kurfürstendamm ruft die Bundesregierung mit Hilfe von Leuchtreklamen zu Abstand und Maskentragen auf. Es erscheinen Porträts von Bürger*innen, denen Sätze in den Mund gelegt werden, die zu Kontaktreduktion und -monitoring motivieren.[1] Wie können solche Bilder angesichts einer hochansteckenden Krankheit wie Covid-19 aus stadtsoziologischer Sicht gedeutet werden? Welche Perspektive auf Stadt machen die derzeitigen Entwicklungen und Krisenerzählungen dringlich? Haben wir es mit einer „planetarischen Enturbanisierung“ zu tun, wie Stefan Höhne und Boris Michel (2021) nahelegen?

Die aktuelle Gesundheitskrise zeigt die Diskrepanz zwischen den Sichtweisen auf gesellschaftliches Zusammenleben, wie sie sich in offiziellen Politiken ausdrücken, und dem städtischen Alltag der Vielen (Blokland/Feltran i. E.). Diese Vielen beschreiben keine demografische und politische Mehrheit, sondern verweisen, in Anlehnung an AbdouMaliq Simones Konzept der „urban majority“ (ebd. 2013), auf improvisierte Formen städtischen Lebens heterogener Akteur*innen, die weder zur städtischen Mittelschicht noch zu den am stärksten Marginalisierten gehören. Dieses „kollektive Leben“ (Bhan et al. 2020) einer „Stadt im Entstehen“ (Simone 2010) konstituiert lokale urbane Welten, die über sich hinausweisen, aber als situative und situierte Figurationen von Akteur*innen, Dingen und Räumen untersucht werden können.

Die Stadtforschung sollte die Diskrepanz zwischen dem Alltag der Vielen und den Sichtweisen offizieller Politiken (Blokland/Feltran i. E.) zum Ausgangspunkt ihres Nachdenkens über das Urbane machen. Dadurch könnte verhindert werden, dass Sozialforschung abstrakte Konzepte und 
Symbole reproduziert, mit denen Gesellschaft regiert wird, indem sie die Tatsachen des Lebens und die Erfahrungen der Menschen in eine konzeptuelle Währung überträgt, mit der sie regiert werden können (Smith 1990). Die neun Symptome einer Krise des Städtischen angesichts der Covid-19Pandemie, die Höhne und Michel (2021) herausarbeiten, sind zu Teilen auch symptomatisch für eine Problematisierung und Vorstellung des Städtischen, die zum einen auf fixierte Einheiten (wie Familie, Arbeitsplatz oder Wohnort) bauen und zum anderen Problembereiche kennzeichnen, die eher für eine kleine Gruppe von Stadtbewohner*innen relevant sind. Anders formuliert: Wenn wir das Städtische ausgehend von der Einheit des Haushalts, vom Büro oder vom Erwerb von Eigentum (das ,Haus im Grünen') denken, verlieren wir die gelebten Verbindungen und städtischen Zwischenräume aus dem Blick, die konstitutiv für das Urbane sind - nicht nur, aber gerade aus dem Standpunkt der Vielen. Die Organisation des Lebensunterhalts umfasst ökonomische Aktivitäten jenseits des Büros oder der Fabrik. Sie basiert auf dem Ineinandergreifen heterogener Praktiken und Akteur*innen. Städtisches Leben ist von Verletzlichkeit geprägt, es bedarf der Unterstützung durch andere in vielfältigen Konfigurationen - auch jenseits einer heterosexuellen Kernfamilie. Politische Maßnahmen sind weniger Ausgangspunkt oder Hintergrund für eine Sezierung der Krise des Städtischen. Stattdessen ist das konkrete, erlebte staatliche Handeln selbst Teil des Städtischen. Im Folgenden plädiere ich für ein Nachdenken über Urbanität angesichts der Covid-19-Pandemie aus genau solch einem Standpunkt der Vielen. Zwei Regulierungsfelder aktueller Coronapolitiken, die Arbeitszeit und die Kontaktbeschränkungen, dienen mir dabei als Ausgangspunkt.

In der Diskussion über das Ende des Städtischen steht die Verlagerung von Arbeit ins Homeoffice im Mittelpunkt (vgl. u. a. Overmann/Nathan 2020). Ohne die damit verbundenen Herausforderungen, etwa die Neuordnungen von Sorge-Arrangements, zu negieren, erscheint mir dieser Fokus jedoch zu kurz gegriffen. Aus einer Sicht der Vielen drängt sich vielmehr die Frage auf, wie aktuelle Einschränkungen von Kulturangeboten, Gastronomie und Einzelhandel die Organisation des Lebensunterhalt jener Stadtbewohner*innen beeinflusst, die keine feste (Vollzeit-)Anstellung haben oder in sogenannten systemrelevanten Berufen weiterhin ihrer Tätigkeit nachgehen müssen. Erste Studien zeigen, dass die Anzahl der Minijobber*innen in Deutschland rasant abgenommen hat (Grabka/Braband/Göbler 2020). Bei Menschen ohne beruflichen Abschluss war die Wahrscheinlichkeit, dass sie ihre Tätigkeit unterbrochen haben, in den letzten Monaten größer (Bünning/Hipp/Munnes 2020). In Deutschland (Koebe et al. 2020), aber auch international scheint die offizielle Kategorisierung nach Systemrelevanz vor allem die Produktivität aufrechtzuerhalten. Die systemrelevanten Arbeiter*innen selbst bleiben oftmals in prekären Einkommenslagen und mit Gesundheitsrisiken konfrontiert (Stevano/Ali/Jamieson 2020). Stadtforschung muss sich mehr denn je mit diesen Prekarisierungsprozessen auseinandersetzen, die in der Covid-19-Pandemie sichtbar bzw. verstärkt werden. Das beginnt mit der Frage, welche Tätigkeiten, welche Orte und welche Akteur*innen wir in die Diskussion über das ökonomische Leben in Städten miteinbeziehen. Dabei sollten wir über die Lohnarbeit hinausgehen und die Multiplizität ökonomischer Aktivitäten von Stadtbewohner*innen 
beachten, auch in empirischen Analysen und Theorien zum Urbanen. Stadtbewohner*innen organisieren sich nicht nur im sogenannten Globalen Süden in diversen Ökonomien, die nicht zwangsläufig staatlich vermessen werden (Schilling 2020; Gibson-Graham 2008; Smith/Stenning 2006). Vom Standpunkt des Alltags der Vielen aus betrachtet steht hinter dem Leerstand von Büros also noch viel mehr, das es zu untersuchen gilt: Leere Büros verändern die sozialen Infrastrukturen derjenigen, die auf der Straße Regenschirme für Büroangestellte verkaufen, sie machen Taxifahrer*innen arbeitslos, die sonst den Weg zum Büro ermöglichen - und andererseits gibt es weiterhin den Sicherheitsdienst und die Reinigungskraft, die tagtäglich in den Büros tätig sind und nebenbei zusätzlich die Betreuung ihrer Kinder organisieren müssen, die nicht zur Schule gehen können.

Auch ein Verständnis des Haushalts als Einheit steht einer Perspektive der Verflechtung ökonomischer Praktiken entgegen. In der Pandemie wurde das Konzept des Haushalts politisiert, indem es zur Maßeinheit für Kontaktbeschränkungen erklärt wurde. Anstatt anzunehmen, dass sich Menschen in die zeitlose und stabile Einheit des Haushalts zurückziehen, der mit Familie gleichgesetzt wird, könnten wir genauer hinschauen und die Aushandlung von Intimität und Nähe, in der Verwandtschaftsbeziehungen aktualisiert werden, sowie die Organisation materieller und emotionaler Unterstützung (Blokland/Vief/Krüger 2020) als Ausgangspunkt nehmen, um über das Städtische nachzudenken. Wie und in welchen Situationen wird die Maßeinheit Haushalt in alltäglichen Praktiken relevant? Sie kann zum Beispiel eine Orientierungsgröße für die Regulierung sozialer Beziehungen sein, das heißt für die (Neu-)Justierung bzw. Verstetigung von Zugehörigkeit und der Rechte auf Nähe und Unterstützung. Daran könnte sich anschließen, zu untersuchen, wie unterschiedlich Haushaltsdisziplinierung verschiedene Bewohner*innen und Wohnarrangements trifft; welche sozialen Konstellationen von „street level bureaucrats“ (Lipsky 1980) und Stadtbewohner*innen als Haushalt gelesen werden bzw. wer wo verdächtigt und sanktioniert wird. Der vermeintlich private Raum entsteht erst in Abgrenzung vom Außen, dem Städtischen, dem ,vor der Haustür'. In einem ähnlichen Zusammenhang wird auch Familie als „heiliger Raum“ (Bourdieu 1993) konstituiert und stets neu aktualisiert.

Genauso wenig wie Familie und Haushalt als gegebene Basiseinheiten vorausgesetzt werden können, existieren Stadtbewohner*innen als individuelle Essenzen. Anders als die eingangs erwähnte Kampagne \#fürmichfüruns der Bundesregierung suggeriert, sind wir eben keine unabhängigen Akteur*innen, die stabile Motivationen und Anreize haben, nach denen wir situationsübergreifend konsistent handeln. Vielmehr weben wir unsere soziale Existenz in sozialen Beziehungen und Netzwerken, in denen Regeln je nach Situation und sozialem Kontext genutzt werden, um Zugehörigkeit zu bekräftigen, Mitgliedschaft zu regulieren sowie Ehre und Reputation zu organisieren. Risikoberechnung wird so zu einer weit komplexeren Angelegenheit als das bloße Zählen von Infektionszahlen und Kontaktpersonen: Es zählt auch der Verlust von Zugehörigkeit, von Anerkennung, von sozialer Existenz! So kann es in einer Situation wichtig sein, eine Maske zu tragen, um so den Respekt für andere zu vermitteln. In einer anderen Situation jedoch kann das Ablegen der Maske ein Zeichen von Nähe sein. Die Symbolpolitik der Maske, 
die situierte Bedeutung von Regeln und ihrer Umgehung in Analysen miteinzubeziehen, kann dabei helfen, Dynamiken der Ausgrenzung, Vereinsamung oder Exposition gegenüber Gesundheitsrisiken im städtischen Alltag zu erklären und aufzuzeigen.[2] Diese Perspektive denkt und erforscht das Städtische aus der Perspektive einer grundsätzlichen Verletzlichkeit, in die menschliche und nicht-menschliche Existenz eingebettet ist (Butler 2006). Vulnerabilität spielt auch im städtischen Alltag eine wichtige Rolle - nicht als Problem, das mittels bestimmter Indikatoren gemessen und verwaltbar gemacht werden kann, sondern als Existenzbedingung, die in der Aushandlung sozialer Interdependenzen sichtbar wird.

Die Politiken der Systemrelevanz und Haushaltsdisziplinierung zeigen die Diskrepanz zwischen einem ,conceptual mode of governance“ und einem städtischen Alltag oder, anders formuliert, das Zusammen-bzw. Gegenspiel von „institutional logic“ und „everyday logic“ (Blokland/Feltran i. E.). Dieses Scharnier ist auch in der aktuellen Krise von großer Bedeutung und rückt staatliches Handeln als analytische Perspektive weiter ins Blickfeld der Stadtforschung (Hilbrandt 2019). In der Art und Weise, wie staatliche Praxis in der aktuellen Gesundheitskrise um Covid-19 im städtischen Alltag von Bewohner*innen westeuropäischer Großstädte wie Berlin präsent wird, können wir viel von der Forschung zu Städten des Südens lernen. Voraussehbarkeit und Reliabilität staatlichen Handeln ist in diesen Forschungskontexten selten und keinesfalls ein zentraler Referenzpunkt für die Organisation nachhaltiger Lebensbedingungen der Vielen. Das eröffnet auch neue theoretische Perspektiven auf urbane Ordnung (Jeffrey/ Dyson 2013; Mbembe/Roitman 1995).

Auf der anderen Seite können die institutionellen Logiken selbst weiter untersucht werden: Sie sind von Kategorisierungen geprägt, mit denen empirische Realitäten lesbar und damit auch verwaltbar gemacht werden können (Fourcade/Gordon 2020; Scott 1998). Im gegenwärtigen Krisenmanagement (europäischer) Staaten zeigt sich, wie Regierungsakteur*innen versuchen, eine Krisensituation zu managen und auf Grundlage von Echtzeitdaten temporär zu verwalten: Tägliche Inzidenzen, Todesfälle, Reproduktionszahlen und Intensivbetten-Belegungen sind allesamt statistische Kennzahlen, die ins Zentrum gerückt und als Legitimationsgrundlage für politisches Handeln herangezogen werden. Systemische Missstände und Verkettungen von Umständen, die eine Krisensituation überhaupt erst entstehen lassen, werden wenig thematisiert, geschweige denn verändert.

Diese Präsenz des Staates verweist auf den Einfluss der Tech-Branche auf staatliches Handeln. Das Sammeln von Daten nutzen staatliche Akteur*innen für die Anpassung und Modellierung von Verhalten in Echtzeit. Politiken sind an Modellberechnungen und Voraussagen orientiert, um kurzfristig auf Situationen reagieren zu können (Fourcade/Gordon 2020). Das Vorgehen standardisiert menschliches Handeln und verkürzt soziale Probleme auf ein Problem, das scheinbar mithilfe von Technologie gelöst werden kann und sollte. Diese Philosophie des Solutionismus ist eng verknüpft mit technologischen Entwicklungen und Unternehmenspolitiken des Silicon Valley (Nachtwey/Seidl 2020): In der Erforschung der ökonomischen Restrukturierung und der politischen Ökonomie von Post-CoronaStädten wird uns diese ideologische und materielle Partnerschaft zwischen 
Staat und Digitalkonzernen weiter beschäftigen. Schon jetzt zeichnet sich ab, dass Digitalkonzerne die großen Gewinner der Krise sind - und dabei den städtischen Raum mitprägen: als Logistik-Infrastrukturen, Kommunikationswerkzeuge und Dienstleistungsanbieter. Wenn wir diesen Trend nicht thematisieren und ihn nicht in der Diskussion über das Städtische aufgreifen, verpassen wir, wie diese Konzerne Grundlagen für ökonomische Sicherheit und lokale urbane Welten in Städten mitgestalten.

Mit diesen drei Zugangsebenen zum Städtischen, der Verletzlichkeit und Interdependenz, der Multiplizität von Arbeit und der Präsenz von Staatshandeln, könnte ein Gegengewicht zur Erzählung vom Ende des Städtischen als Privatisierung, Landflucht und Vereinzelung gebildet werden. Besser gesagt: Wir können diese Erzählung anders mit Bedeutung füllen. Am Ende steht kein ,ja' oder ,nein' zur Enturbanisierung, sondern eine Analyse, inwieweit diese Frage inhärenter Teil einer Krisenerzählung ist und welche sozialen Dynamiken der lokalen urbanen Welt darin eingeschlossen sind, aber nicht als solche benannt werden.

Dieser Artikel wurde durch Mittel des Open Access Publikationsfonds der Friedrich-Schiller-Universität Jena gefördert.

\section{Endnoten}

[1] Zwei dieser Sätze sind „Ich will, dass unsere Kita offen bleibt. Dafür nutze ich jetzt die Corona-Warn-App.“ und „Ich will wieder tanzen. Dafür fahr ich jetzt Kontakte runter.“ (vgl. BMG 2021).

[2] Vgl. das Forschungsprojekt „Getting things done in a city on hold“ unter Leitung von Talja Blokland (HU Berlin) und Johanna Hoerning (TU Berlin).

\section{Autor innen}

Hannah Schilling ist Stadtsoziologin und arbeitet zu städtischen Ökonomien und sozialer Ungleichheit in globaler Perspektive. Sie promovierte mit einer vergleichenden Analyse von Prekarisierungsprozessen unter jungen Erwachsenen in digitaler Arbeit in Abidjan und Berlin. hannah.schilling@uni-jena.de

\section{Literatur}

Bhan, Gautam / Caldeira, Teresa / Gillespie, Kelly / Simone, AbdouMaliq (2020): The pandemic, southern urbanisms and collective life. In: Society and Space, 3.8.2020. https://www.societyandspace.org/articles/the-pandemic-southern-urbanisms-andcollective-life (letzter Zugriff 21.2.2021).

Blokland, Talja / Feltran, Gabriel de Santis (i. E.): Impasse: How institutional and everyday logics reproduce urban inequalities (special issue). In: Tijdschrift voor Economische en Sociale Geografie.

Blokland, Talja / Vief, Robert / Krüger, Daniela (2020): Leaving the house to talk in private. How COVID19 restrictions affected how and where we find someone to talk to. In: SFB $1265 \mathrm{Blog}, 21.12 .2021 . \mathrm{https} / / / \mathrm{sfb} 1265 . \mathrm{de} / \mathrm{blog} / \mathrm{leaving}$-the-house-to-talk-in-privatehow-covid19-restrictions-affected-how-and-where-we-find-someone-to-talk-to/ (letzter Zugriff 07.03.21).

Bourdieu, Pierre (1993): À propos de la famille comme catégorie réalisée. In: Actes de la Recherche en Sciences Sociales 100/1, 32-36. 
BMG - Bundesministerium für Gesundheit (2021): \#FürMichFürUns. https://www.zusammengegencorona.de/fuermichfueruns/ (letzter Zugriff am 19.1.2021).

Bünning, Mareike / Hipp, Lena / Munnes, Stefan (2020): Erwerbsarbeit in Zeiten von Corona. WZB Ergebnisbericht. Berlin: Wissenschaftszentrum Berlin für Sozialforschung.

Butler, Judith (2006): Precarious life: The powers of mourning and violence. London/New York: Verso.

Fourcade, Marion / Gordon, Jeffrey (2020): Learning like a state. Statecraft in the digital age. In: Journal of Law and Political Economy 1/1, 78-108.

Gibson-Graham, Julie Katherine (2008): Diverse economies. Performative practices for other worlds. In: Progress in Human Geography 32/5, 613-632.

Grabka, Markus / Braband, Carsten / Göbler, Konstantin (2020): Beschäftigte in Minijobs sind VerliererInnen der coronabedingten Rezession. In: DIW Wochenbericht 45, 841-847. https://www.diw.de/de/diw_o1.c.802083.de/publikationen/wochenberichte/2020_45_1/beschaeftigte_in_minijobs_sind_verliererinnen_der_coronabedingten_rezession.html (letzter Zugriff am 20.1.2021).

Hilbrandt, Hanna (2019): Everyday urbanism and the everyday state. In: Urban Studies $56 / 2,352-367$.

Lipsky, Michael (1980): Street-level bureaucracy. Dilemmas of the individual in public services. New York: Sage.

Höhne, Stefan / Michel, Boris (2021): Das Ende des Städtischen? Pandemie, Digitalisierung und planetarische Enturbanisierung. In: sub \urban. zeitschrift für kritische stadtforschung 9/1-2, 141-149.

Jeffrey, Craig / Dyson, Jane (2013): Zigzag capitalism. In: Geoforum 49, R1-R3.

Koebe, Josefine / Samtleben, Claire / Schrenker, Annekatrin / Zucco, Aline (2020): Systemrelevant, aber dennoch kaum anerkannt: Entlohnung unverzichtbarer Berufe in der Corona-Krise unterdurchschnittlich. In: DIW aktuell 48. https://www.diw.de/de/ diw_01.c.792754.de/publikationen/diw_aktuell/2020_0048/systemrelevant_aber_ dennoch_kaum_anerkannt_entlohnung_unverzichtbarer_berufe_in_der_coronakrise_unterdurchschnittlich.html (letzter Zugriff am 20.1.2021).

Mbembe, Achille / Roitman, Janet (1995): Figures of the subject in times of crisis. In: Public Culture 7/2, 323-352.

Nachtwey, Oliver / Seidl, Timo (2020): The solutionist ethic and the spirit of digital capitalism. In: SocArXiv, https://doi.org/10.31235/osf.io/sgizq.

Overman, Henry / Nathan, Max (2020): Will coronavirus cause a big city exodus? In: LSE COVID-19 blog, 10.12.2020. https://blogs.lse.ac.uk/covid19/2020/12/10/will-coronavirus-cause-a-big-city-exodus/ (letzter Zugriff am 20.1.2021).

Schäfer, Kristina Antonia (2020): Welche Corona-Immobilien-Mythen stimmen. Preissturz, Angebotseinbruch, Stadtflucht? In: Wirtschaftswoche Online vom 4.6.2020. https:// www.wiwo.de/finanzen/immobilien/preissturz-angebotseinbruch-stadtflucht-welchecorona-immobilien-mythen-stimmen/25886614.html (letzter Zugriff am 19.1.2021).

Schilling, Hannah (2020): Aller se chercher. Dissertation. Berlin: Humboldt Universität zu Berlin.

Scott, James (1998): Seeing like a state. Yale: Yale University Press.

Simone, AbdouMaliq (2010): City life from Jakarta to Dakar. New York: Routledge.

Simone, AbdouMaliq (2013): Cities of uncertainty. Jakarta, the urban majority, and inventive political technologies. In: Theory, Culture \& Society 30/7-8, 243-263.

Smith, Adrian / Stenning, Alison (2006): Beyond household economies. Articulations and spaces of economic practice in postsocialism. In: Progress in Human Geography 30/2, 190-213.

Smith, Dorothy (1990): The conceptual practices of power. Boston: Northeastern University Press.

Stevano, Sara / Ali, Rosimina / Jamieson, Merle (2020): Essential for what? A global social reproduction view on the re-organisation of work during the COVID-19 pandemic. In: Canadian Journal of Development Studies, https://doi.org/10.1080/02255189.2020.18 34362.

Welt auf Abstand. Reise durch ein besonderes Jahr (2020): ZDF, 89:0o Minuten. https:// www.arte.tv/de/videos/o98771-ooo-A/welt-auf-abstand/ (letzter Zugriff am 19.1.2021). Cristina Trebbi / Jobst Knigge (Buch und Regie). 